\title{
Retinal Vascular Geometry Predicts Incident Retinopathy in Young People With Type 1 Diabetes
}

Paul Benitez-Aguirre, MPH, DCH, FRACP ${ }^{1,2}$ Maria E. Craig, fracP, PhD ${ }^{1,2,3}$

Muhammad Bayu SasongKo, MD, mepi ${ }^{4,5}$ Alicia J. Jenkins, MD ${ }^{6}$
TIEN YIN WONG, MD, PHD ${ }^{4,7}$ JiE Jin WANG, MMED, PHD ${ }^{4,8}$

Ning Cheung, mBbs 4

Kim C. Donaghue, FRACP, PhD ${ }^{1,2}$

OBJECTIVE_-To examine the association between retinal vascular geometry and subsequent development of incident retinopathy in young patients with type 1 diabetes.

RESEARCH DESICN AND METHODS - A prospective cohort study of 736 people with type 1 diabetes aged 12 to 20 years, retinopathy-free at baseline, attending an Australian tertiary care hospital. Retinopathy was determined from seven-field retinal photographs according to the modified Airlie House Classification. Retinal vascular geometry, including length/diameter ratio (LDR) and simple tortuosity (ST), was quantified in baseline retinal photographs. Generalized estimating equations were used to determine risk of retinopathy associated with baseline LDR and ST, adjusting for other factors.

RESULTS-After a median 3.8 (interquartile range 2.4-6.1) years of follow-up, incident retinopathy developed in 287 of 736 (39\%). In multivariate analysis, lower arteriolar LDR (odds ratio 1.8 [95\% CI 1.2-2.6]; 1st vs. 4th quartile) and greater arteriolar ST (1.5 [1.0-2.2]; 4th vs. lst quartile) predicted incident retinopathy after adjusting for diabetes duration, sex, AlC, blood pressure, total cholesterol, and BMI. In subgroup analysis by sex, LDR predicted incident retinopathy in male and female participants (2.1 [1.1-4.0] and 1.7 [1.1-2.7]; 1st vs. 4th quartiles, respectively) and greater arteriolar ST predicted incident retinopathy in male participants (2.4 [1.1-4.4]; 4th vs. 1st quartile) only.

CONCLUSIONS _ Lower arteriolar LDR and greater ST were independently associated with incident retinopathy in young people with type 1 diabetes. These vascular geometry measures may serve as risk markers for diabetic retinopathy and provide insights into the early structural changes in diabetic microvascular complications.

Diabetes Care 34:1622-1627, 2011

$\mathbf{T}$ he retina offers a unique opportunity to noninvasively and repeatedly examine the microvasculature in vivo. Improved imaging techniques and advances in computer-based retinal image analysis have allowed a better understanding of retinal vascular parameters and their

relationship with pathophysiologic processes in and beyond the retina $(1,2)$. Diabetic retinopathy (DR) is the most common microvascular complication in type 1 diabetes and is a risk indicator of other complications, with mild retinopathy being associated with several-fold

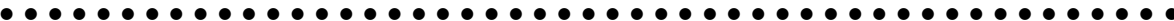

From ${ }^{1}$ The Children's Hospital at Westmead, Westmead, New South Wales, Australia; the ${ }^{2}$ Discipline of Paediatrics and Child Health, The University of Sydney, Sydney, New South Wales, Australia; the ${ }^{3}$ School of Women's and Children's Health, University of New South Wales, Sydney, New South Wales, Australia; the ${ }^{4}$ Centre for Eye Research Australia, Royal Victorian Eye and Ear Hospital, University of Melbourne, Melbourne, Victoria, Australia; the ${ }^{5}$ Department of Ophthalmology, Faculty of Medicine, Gadjah Mada University, Yogyakarta, Indonesia; the ${ }^{6}$ Department of Medicine, St. Vincent's Hospital, University of Melbourne, Melbourne, Victoria, Australia; the ${ }^{7}$ Singapore Eye Research Institute, National University of Singapore, Singapore; and the ${ }^{8}$ Centre for Vision Research, University of Sydney, Sydney, New South Wales, Australia.

Corresponding author: Kim C. Donaghue, kimd@chw.edu.au.

Received 23 December 2010 and accepted 14 April 2011

DOI: $10.2337 / \mathrm{dcl} 0-2419$

(C) 2011 by the American Diabetes Association. Readers may use this article as long as the work is properly cited, the use is educational and not for profit, and the work is not altered. See http://creativecommons.org/ licenses/by-nc-nd/3.0/ for details.

increases in the risk of stroke, coronary heart disease, and heart failure independent of other risk factors (3). A better understanding of DR may allow prevention and early treatment of those at risk for vision loss and life-threatening cardiovascular disease.

Hypothetically, optimal retinal microvascular architecture will deliver the most efficient blood flow (4). Alterations in retinal architecture may reflect early vascular dysfunction, concurrent compensatory mechanisms, and predict later disease (5). Indeed, subtle variations in retinal vessel caliber have been associated with cardiovascular risk factors (6) and, more recently, diabetic retinopathy and nephropathy $(7,8)$. Some diabetes studies, however, have highlighted the variable nature of retinal caliber with ambient glycemia (9). Thus, geometric parameters, such as vessel simple tortuosity (ST), which reflects the undulation of a vessel along its course, and length/diameter ratio (LDR), which uses the axial length between two defined branching points divided by the vessel's diameter, may be more robust predictors of diabetic microvascular and macrovascular complications.

We previously demonstrated in a cross-sectional analysis that an adverse diabetes risk profile (higher AlC and systolic blood pressure) is associated with lower retinal arteriolar LDR and greater tortuosity in young people with type 1 diabetes (10). We therefore hypothesized that changes in LDR and ST might predict incident retinopathy and conducted this study to prospectively assess the association between retinal vascular geometric parameters (LDR and ST) and incident retinopathy in young patients with type 1 diabetes.

\section{RESEARCH DESICN AND METHODS}

Study population and assessment A total of 951 adolescents aged between 12 and 20 years with type 1 diabetes of at least 2 years' duration, who were 
retinopathy-free at baseline and had a minimum of two assessments through the Diabetes Complications Assessment Service at the Children's Hospital at Westmead between 1990 and 2007, agreed to participate in the study. All participants had at least a 2-year study follow-up from the baseline visit. Of these, 736 (77\%) had baseline photographs suitable for retinal vascular geometry grading and were included in this longitudinal study. The hospital ethics committee approved the study, and written informed consent was obtained from all participants.

Participants were prospectively followed up using standardized interviews, clinical examinations, and laboratory investigations at baseline and at subsequent visits, as previously described (10). Briefly, BMI $\left(\mathrm{kg} / \mathrm{m}^{2}\right)$ was assessed and blood pressure (BP) was measured with a sphygmomanometer using an appropriately sized cuff in seated patients after 5 min of rest. Venous blood samples were obtained for measurement of AlC and total cholesterol levels.

\section{Definition of retinopathy}

Mydriatic seven-field stereoscopic fundal photography of both eyes was performed using a TRC 50-VT Topcon Fundus Camera (Tokyo Optical, Tokyo, Japan), initially with film and then with digital photography (11) beginning in September 2004. Camera settings, including angle of retinal photography, remained unchanged. All film slides before September 2004 were digitized using a CanoScan FS2710 (Canon, Tokyo, Japan) film scanner, set to automatic exposure and focus, with 24-bit color and a maximum resolution of 2,720 dpi without enhancement. These digitized images were used to assess baseline retinal vascular geometry.

Retinopathy was assessed and graded from these images by a single ophthalmologist masked to participants' clinical characteristics, according to the Early Treatment Diabetic Retinopathy Study (ETDRS) adaptation of the modified Airlie House classification (12). Incident retinopathy was defined as at least one microaneurysm/hemorrhage in either eye (ETDRS level 21, minimal nonproliferative diabetic retinopathy or greater).

\section{Retinal vascular geometry measurements}

Retinal vascular geometry was retrospectively analyzed from baseline retinal photographs. All slides were individually assessed for grading suitability. We considered images to be of poor quality and ungradable if they were blurred, did not completely represent all zones, or if there were fewer than four large arterioles or venules gradable in one image. Right eye digitized retinal photographs of each patient were analyzed by a single grader, masked to participants' characteristics, using a semiautomated computer-assisted image program (Singapore I Vessel Assessment [SIVA], The National University of Singapore) as previously described (10). This software is able to detect the optic disc and divide the image into three concentric zones: zone $\mathrm{A}$, within 0.5-disc diameter from the optic disc margin; zone B, 0.5- to 1.0-optic disc diameter away; and zone C, 1.0- to 2.0-disc diameter away.

Once the optic disc and zones were identified, vessels were traced. Retinal vessel caliber measurements, represented by the central retinal arteriolar equivalent (CRAE) and the central retinal venular equivalent (CRVE), were calculated as previously described. Measurements were based on the biggest six arterioles and six venules within zone $B$ and $C$. The grader ensured that the vessel type selected by the program was correct. The software then combined the individual measurements into summary indices:

- LDR was calculated as the length from the midpoint of the first branch to the midpoint of the second branch divided by the diameter of the parent vessel at the first branch.

- ST was calculated as the ratio between the actual path length of the vessel segment (measured by tracking) and the straight-line length of the same segment.

\section{Statistical analysis}

All statistical procedures were performed using PASW 18.0 software (SPSS Inc., Chicago, IL). Descriptive statistics are presented as mean and SD for normally distributed data or median and interquartile range (IQR) for skewed distributions. Differences between continuous independent samples were evaluated by using the Student $t$ test for normally distributed data, or the Mann-Whitney U test or the Kruskal-Wallis test for skewed data. ANOVA was used to determine differences in LDR quartile groups with the Tukey test for post hoc analysis. Differences between groups of categoric variables were compared using the $\chi^{2}$ test.

Longitudinal analysis was performed using generalized estimating equations
(GEE), with presence or absence of retinopathy at the respective visit as the outcome variable. The use of GEE allowed all visits for each individual to be included in the analysis and accounted for correlations between repeated observations for a given patient. Age; sex; diabetes duration; AlC; systolic BP (SBP); diastolic BP (DBP); BMI SD scores (SDS); total cholesterol; and albumin excretion rate (AER), expressed as log AER, were included in the models as covariates. Baseline arterial and venular ST and LDR values were log-transformed for analysis as continuous variables. ST and LDR were categorized into quartiles to examine for threshold effects. Clinically relevant interaction terms were included in the models. Analysis was stratified by sex in view of sex differences in vessel calibers. Results of significant GEE models are reported as odds ratio (OR) and 95\% CI

RESULTS - Baseline characteristics are reported in Table 1. Median age was 13.7 (IQR 12.9-15.1) years and diabetes duration was 5.0 (3.3-7.6) years. After a median 3.8 (2.4-6.1) years of follow-up with 3.0 (2.0-4.0) visits per participant and 2,745 clinical assessments, diabetic retinopathy developed in 287 of 736 participants (39.0\%). Those who developed incident retinopathy were generally younger, had a longer diabetes duration, had higher AlC levels, had higher BP, and were less likely to be following a regimen of multiple daily insulin injections (Table 1). A higher proportion of females developed retinopathy compared to male subjects ( 42.1 vs. $35.8 \%, P=0.08$ )

The lowest quartile for arteriolar LDR (LDRa) had a greater proportion of individuals with incident retinopathy, higher $\mathrm{AlC}$, higher SBP and DBP, and fewer patients receiving intensive insulin therapy (Table 1). Arteriolar caliber did not differ among LDRa quartiles in male patients $\left(F_{(3,354)}=0.67, P=0.6\right)$. Arteriolar caliber differed among LDRa quartiles in female patients $\left(F_{(3,374)}=4.68, P=0.003\right)$. Post hoc comparisons among female subjects indicated those in the first and fourth quartile groups had wider arterioles (161.6 and $158.5 \mu \mathrm{m}$, respectively) than those in the second and third LDRa quartiles (154.1 and $156.1 \mu \mathrm{m}$, respectively; $P=0.003)$.

Retinal arteriolar and venular calibers were greater in those with longer $(>5$ years) diabetes duration (158 vs. $154 \mu \mathrm{m}$ $[P=0.001]$ and 220 vs. $215 \mu \mathrm{m}[P=0.004]$, 
Table 1-Baseline characteristics by retinopathy status and LDRa quartiles

\begin{tabular}{|c|c|c|c|c|c|c|c|c|}
\hline \multirow[b]{2}{*}{ Variable } & \multicolumn{3}{|c|}{ Retinopathy* } & \multicolumn{5}{|c|}{ LDRa $\dagger$} \\
\hline & $\begin{array}{c}\text { No } \\
n=449\end{array}$ & $\begin{array}{c}\text { Yes } \\
n=287\end{array}$ & $P$ & $\begin{array}{c}\text { 1st quartile } \\
n=184\end{array}$ & $\begin{array}{c}\text { 2nd quartile } \\
\qquad n=184\end{array}$ & $\begin{array}{c}\text { 3rd quartile } \\
\quad n=184\end{array}$ & $\begin{array}{c}\text { 4th quartile } \\
\qquad n=184\end{array}$ & $P$ \\
\hline \multicolumn{9}{|l|}{ Incident } \\
\hline retinopathy & $\begin{array}{r}0 / 449[0] \\
30 / 440[1] 21\end{array}$ & $\begin{array}{l}287 / 287[100] \\
128 / 287[446]\end{array}$ & 088 & $\begin{array}{l}92 / 184[50.0] \\
91 / 184[405]\end{array}$ & $\begin{array}{l}68 / 184[37.0] \\
06 / 184[527\end{array}$ & $\begin{array}{l}71 / 184[38.6] \\
87 / 184[473]\end{array}$ & $\begin{array}{l}56 / 184[30.4] \\
84 / 184[457]\end{array}$ & 0.002 \\
\hline Age (years) & $13.9(13.0-15.2)$ & $13.4(12.7-14.8)$ & 0.006 & $13.4(12.8-14.8)$ & $13.7(12.8-15.2)$ & $13.7(12.8-15.1)$ & $13.9(12.9-15.0)$ & 0.4 \\
\hline \multicolumn{9}{|l|}{$\begin{array}{l}\text { Diabetes } \\
\text { duration }\end{array}$} \\
\hline (years) & $4.4(3.0-7.2)$ & $5.3(3.8-8.6)$ & 0.005 & $5.4(3.6-7.9)$ & $5.0(3.2-8.2)$ & $4.6(3.2-7.4)$ & $4.9(2.9-7.4)$ & 0.3 \\
\hline $\mathrm{AlC}(\%)$ & $8.3(1.2)$ & $8.8(1.3)$ & $<0.001$ & $8.7(1.4)$ & $8.5(1.2)$ & $8.3(1.3)$ & $8.5(1.3)$ & 0.02 \\
\hline SBP SDS & $0.15(0.99)$ & $0.32(0.93)$ & 0.03 & $0.46(0.90)$ & $0.21(0.91)$ & $0.15(1.04)$ & $0.05(0.99)$ & 0.001 \\
\hline DBP SDS & $0.38(0.79)$ & $0.47(0.79)$ & 0.2 & $0.56(0.81)$ & $0.42(0.79)$ & $0.33(0.80)$ & $0.35(0.75)$ & 0.02 \\
\hline BMI SDS & $0.64(0.76)$ & $0.52(0.81)$ & 0.05 & $0.63(0.83)$ & $0.58(0.70)$ & $0.59(0.81)$ & $0.57(0.81)$ & 0.9 \\
\hline \multicolumn{9}{|l|}{ Cholesterol } \\
\hline$(\mathrm{mmol} / \mathrm{L})$ & $4.2(3.7-4.7)$ & $4.3(3.7-4.8)$ & 0.6 & $4.2(3.7-4.7)$ & $4.1(3.6-4.6)$ & $4.3(3.8-4.7)$ & $4.3(3.8-4.9)$ & 0.1 \\
\hline LogAER & $0.71(0.33)$ & $0.68(0.27)$ & 0.2 & $0.71(0.31)$ & $0.72(0.32)$ & $0.71(0.31)$ & $0.65(0.28)$ & 0.2 \\
\hline MDI & $117 / 433$ [27.0] & 47/281 [16.7] & 0.001 & 30/179 [16.8] & 43/183 [23.5] & 40/177 [22.6] & $51 / 175[29.1]$ & 0.05 \\
\hline
\end{tabular}

Normal data are depicted as mean (SD), skewed data as median (IQR), and proportions as $n$ [\%]. MDI, multiple daily injections. *Comparison between retinopathy groups used the Student $t$ test for normal data, Mann-Whitney $U$ test for skewed data, and $\chi^{2}$ test for proportions. †Comparisons between LDRa groups used ANOVA for normal data and the Tukey test for multiple comparisons. The Kruskal-Wallis test was used for non-normal data comparisons and $\chi^{2}$ test for proportions.

respectively) and in female versus male patients (arterioles: 158 vs. $154 \mu \mathrm{m}[P<$ $0.001]$; venules: 220 vs. $215 \mu \mathrm{m}[P<$ $0.001]$ ).

\section{Prospective analysis}

In univariate analysis, lower LDRa (OR 1.9 [95\% CI 1.3-2.7]; 1st vs. 4th quartile) and higher arteriolar ST (STa; 1.6 [1.1-2.3]; 4th vs. 1st quartile) predicted incident retinopathy. Log LDRa (0.71 [0.51-0.98]) and $\log$ STa $\left(\times 10^{3} ; 1.01\right.$ [1.00-1.02]) predicted incident retinopathy as continuous variables. Other significant risk factors for incident retinopathy included longer diabetes duration and higher BP.

In multivariate analysis, lower LDRa (OR 1.8 [95\% CI 1.2-2.7]; 1st vs. 4th quartile) and higher STa (1.6 [1.1-2.4]; 4 th vs. 1st quartile) predicted incident retinopathy after accounting for age, sex, diabetes duration, AlC, diastolic blood pressure, BMI, and total cholesterol (Table 2). Log LDRa and log STa did not reach statistical significance in multivariate analysis for the group as a whole.

In subgroup univariate analysis by sex, lower LDRa predicted incident retinopathy in both male and female participants (OR
1.8 [95\% CI 1.1-2.9] and 2.0 [1.1-3.7], respectively; 1st vs. 4th quartile). Higher STa predicted incident retinopathy in male subjects (2.1 [1.2-3.6]; 4th vs. 1st quartile) with a similar trend observed in female patients (1.3 [0.8-2.2]; 4th vs. 1st quartile). $\log \operatorname{LDRa}(0.58$ [0.40-0.83]) and $\log _{3} \mathrm{STa}\left(\times 10^{3} ; 1.02\right.$ [1.00-1.04]), as continuous variables, predicted incident retinopathy in male patients. A similar trend in female patients did not reach statistical significance (log LDRa: 0.86 [0.48-1.5]; log STa: 1.01 [0.99-1.02]). Higher A1C, cholesterol, and DBP were significant risk factors for retinopathy in

Table 2-LDR and ST predict incident retinopathy in prospective GEE univariate and multivariate models

\begin{tabular}{|c|c|c|c|c|c|c|c|c|}
\hline \multirow[b]{2}{*}{ Variable } & \multirow[b]{2}{*}{$n$} & \multirow[b]{2}{*}{ Pts $(n)$} & \multicolumn{2}{|c|}{ Univariate } & \multicolumn{2}{|c|}{ Multivariate $1^{*}$} & \multicolumn{2}{|c|}{ Multivariate $2 \dagger$} \\
\hline & & & OR (95\% CI) & $P$ & OR $(95 \% \mathrm{CI})$ & $P$ & OR (95\% CI) & $P$ \\
\hline LDRa & 736 & 287 & & & & & & \\
\hline \multicolumn{9}{|l|}{ Quartile } \\
\hline $1(<19.8)$ & 184 & 92 & $1.9(1.3-2.7)$ & 0.001 & $1.8(1.2-2.7)$ & 0.003 & $1.8(1.2-2.6)$ & 0.003 \\
\hline $2(19.8-65.6)$ & 184 & 68 & $1.3(0.9-1.9)$ & 0.2 & $1.3(0.9-2.0)$ & 0.2 & $1.3(0.9-2.0)$ & 0.2 \\
\hline $3(65.6-114.2)$ & 184 & 71 & $1.2(0.8-1.8)$ & 0.3 & $1.3(0.9-2.0)$ & 0.2 & $1.3(0.9-1.9)$ & 0.2 \\
\hline $4(>114.2)$ & 184 & 56 & Reference & & Reference & & Reference & \\
\hline $\mathrm{STa}$ & 736 & 287 & & & & & & \\
\hline \multicolumn{9}{|l|}{ Quartile } \\
\hline $1(<1,091)$ & 184 & 66 & Reference & & Reference & & Reference & \\
\hline $2(1,091-1,107)$ & 184 & 76 & $1.5(1.0-2.2)$ & 0.03 & $1.6(1.1-2.4)$ & 0.02 & $1.6(1.1-2.3)$ & 0.02 \\
\hline $3(1,108-1,127)$ & 184 & 69 & $1.5(1.1-2.2)$ & 0.03 & $1.5(1.0-2.2)$ & 0.03 & $1.6(1.1-2.4)$ & 0.02 \\
\hline $4(>1,127)$ & 184 & 76 & $1.6(1.1-2.3)$ & 0.008 & $1.6(1.1-2.4)$ & 0.02 & $1.5(1.0-2.2)$ & 0.03 \\
\hline
\end{tabular}

Pts, patients. *Model 1 adjusted for age, sex, and diabetes duration. $†$ Model 2 adjusted for model 1 covariates plus AlC, DBP, BMI, total cholesterol, and relevant interactions (sex*AlC and sex*cholesterol). 
male patients, whereas greater diabetes duration was significant in both sexes (Table 3 ).

In multivariate analysis after adjusting for age, diabetes duration, $\mathrm{AlC}$, cholesterol, and BP, lower LDRa predicted incident retinopathy in both male and female patients (2.1 [1.1-4.0] and 1.7 [1.1-2.7], respectively; 1st vs. 4th quartiles) and greater arteriolar STa predicted incident retinopathy in male patients (2.4 [1.1-4.4]; 4th vs. 1st quartile) only. Log LDRa $(0.50[0.33-0.76])$ and log STa (1.03 [1.01-1.05]), as continuous variables, predicted incident retinopathy in male patients only.

CONCLUSIONS - This longitudinal study of initially retinopathy-free young people with type 1 diabetes found that retinal vascular geometry, specifically, lower arteriolar LDR and greater ST, predicted incident retinopathy independent of established risk factors, including diabetes duration, glycemic control, BP, and total cholesterol. Furthermore, there appear to be sex-specific effects in retinal vascular architecture and retinopathy risk factor profiles. In post hoc subgroup analysis, lower LDR was a predictor of incident retinopathy in both sexes. Greater ST predicted incident retinopathy in male patients and a similar trend was observed in female patients.

Lower LDR can result from shorter axial length due to earlier branching points or wider vessel calibers, or both. Our results suggest that axial length was shorter among those with the lowest LDRa because there were no significant differences in arteriolar caliber among LDR quartiles in male subjects and no significant differences in arteriolar diameter between the 1st and 4th LDR quartiles in female participants. We hypothesize that in the diabetic milieu with neuroretinal hypoxia (5), early bifurcation may be a compensatory mechanism to increase vessel density and improve retinal perfusion (perhaps the earliest form of neovascularization). This contrasts with the effect of aging observed in nondiabetic middleaged adults, where higher LDR is associated with the risk of ischemic stroke (13). That is, lower vessel density as a result of aging may contribute to ischemic pathology. The contrasting trends in LDR between young patients with type 1 diabetes and aging nondiabetic individuals suggest that diabetes contributes to microvascular complications by mechanisms independent of aging (e.g., neuroretinal ischemia and hypoxia).

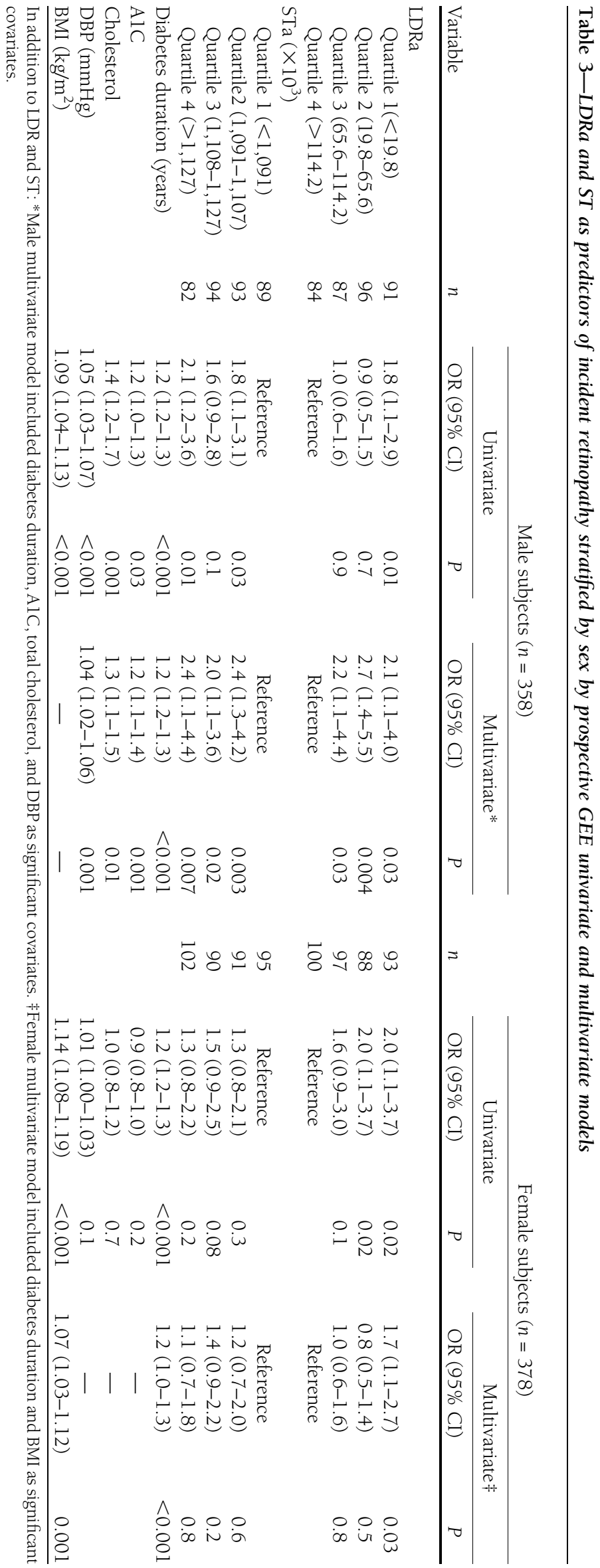


Higher arteriolar tortuosity found among those with subsequent retinopathy may result from compensatory changes in vessel path due to increased vessel density associated with earlier branching and lower LDR. Furthermore, abnormal vessel wall function with loss of tensile forces and increased intramural pressure may alter vessel integrity increasing tortuosity. These findings contrast with an earlier study in which lower arteriolar tortuosity was associated with ischemic heart disease in nondiabetic subjects (13). However, the proposed mechanism of decreased angiogenesis and impaired oxygenation is not inconsistent with our hypothesis. That is, increased tortuosity is, at least in part, an adaptation to increased vessel density (increased angiogenesis) to overcome neuroretinal hypoxia. Thus, increased vessel tortuosity may be a marker of greater angiogenesis in the development of retinopathy.

We previously demonstrated that retinal arteriolar dilatation was associated with retinopathy (7). Our present results, which suggest that vessel caliber is greater in those with longer diabetes duration, are consistent with previous studies suggesting a progression in adults with type 2 diabetes from early vasoconstriction to subsequent vasodilatation with increasing diabetes duration $(14,15)$. Retinal vascular smooth muscle cells are crucial in the regulation of retinal blood flow through the action of large-conductance $\mathrm{Ca}^{2+}$-activated $\mathrm{K}^{+}$channels. These channels show reduced $\mathrm{Ca}^{2+}$ sensitivity during early diabetes, resulting in early sustained vasoconstriction compared with nondiabetic control individuals (16). Subsequent smooth muscle cell and pericyte loss lead to further vasomotor dysregulation, loss of structural support, capillary dropout, and sustained vasodilation (17). This loss in structural integrity of vessels may also contribute to the observed increase in tortuosity.

We previously described the sex dimorphism of greater retinal vascular calibers in girls (7); however, the mechanisms underlying these differences are not clear. In this cohort, a higher proportion of girls developed retinopathy. Girls generally progress through puberty earlier than boys, and the differential observed may represent accelerated retinal vascular changes in girls. There is increasing evidence that puberty and sex hormones may accelerate microvascular complications (18-20), and some have postulated that younger women with diabetes may be more susceptible to macrovascular and microvascular disease than men (21). Our finding that retinopathy developed in those who had an earlier diagnosis of diabetes is consistent with previous findings that longer prepubertal diabetes duration increased the risk of retinopathy (18).

The importance of higher DBP, albeit in the normal range, is highlighted in this study because it remained a significant independent predictor of retinopathy, consistent with our previous findings (22). This suggests that early microvascular changes have organ-specific and systemic hemodynamic effects. The greatest resistance in systemic circulation is offered by capillary beds. Early microvascular changes and dysregulation may increase systemic BP, which in turn may lead to further retinal vascular changes in a selfperpetuating cycle. These findings are consistent with previous studies associating greater arteriolar tortuosity with hypertension in young (23) and older (24) age-groups.

The strengths of this study include the longitudinal design, a large patient cohort with multiple visits per individual, standardized quantitative evaluation of retinal vascular measures by a single grader masked to the clinical status, and the use of GEEs to include every available patient visit in the analysis. Repeated LDR and ST measures at each visit would strengthen this study.

Potential limitations include the differences in length of follow-up and number of visits per individual: our median was 3.8 years, and some patients had only 2 years of follow-up; however, the GEE model accounts for uneven follow-up and missing data. The cohort was from a tertiary referral center and may be biased toward closer monitoring, tighter metabolic control, and a potential bias toward under-representation of retinopathy. However, this attests to the robust nature of the measures studied.

Manual intervention by the grader to ensure correct identification of vessels and their precise location was sometimes required by the semiautomated grading program. This introduced the potential for measurement errors to occur. However, because these measurement errors would occur randomly, the positive associations found in this study are unlikely to be significantly affected.

In summary, retinal vascular geometry, specifically retinal arteriolar LDR and tortuosity, may predict incident retinopathy in young people with type 1 diabetes. These noninvasive retinal measures may aid in elucidating early mechanistic pathways for microvascular complications, assist in risk stratification, and offer a potential to monitor the benefit of therapy early in the course of disease. Further studies replicating these results in other populations are necessary to support these novel findings.

Acknowledgments-The National Health and Medical Research Council (NHMRC 475605) and the Juvenile Diabetes Research Foundation (JDRF 5-2008-274) provided funds for this project.

No potential conflicts of interest relevant to this article were reported.

P.B.-A. digitized photos for grading, analyzed data, and wrote the manuscript. M.E.C. advised on statistical analysis, contributed to the introduction and discussion, and reviewed the manuscript. M.B.S. graded photographs, advised on statistical analysis, and reviewed the manuscript. A.J.J. reviewed the manuscript and contributed to discussion. T.Y.W. contributed to study design and methods and reviewed the manuscript. J.J.W. reviewed the manuscript and contributed to discussion. N.C. contributed to methods and reviewed the manuscript. K.C.D. contributed to the study design, introduction, data analysis, and discussion, and reviewed the manuscript.

The authors are grateful for the support of the participants, their families, and the staff from the Diabetes Complications Assessment Service at The Children's Hospital at Westmead: Janine Cusumano (RN), Alison Pryke (orthoptist), Tracey Jopling (RN), and Jill Brown (volunteer).

\section{References}

1. Hughes AD, Wong TY, Witt N, et al. Determinants of retinal microvascular architecture in normal subjects. Microcirculation 2009;16:159-166

2. Liew G, Wang JJ, Mitchell P, Wong TY. Retinal vascular imaging: a new tool in microvascular disease research. Circ Cardiovasc Imaging 2008;1:156-161

3. Cheung N, Mitchell P, Wong TY. Diabetic retinopathy. Lancet 2010;376:124-136

4. Zamir M. Optimality principles in arterial branching. J Theor Biol 1976;62:227-251

5. Alder VA, Su EN, Yu DY, Cringle SJ, Yu PK. Diabetic retinopathy: early functional changes. Clin Exp Pharmacol Physiol 1997;24:785-788

6. Wong TY, Islam FMA, Klein R, et al. Retinal vascular caliber, cardiovascular risk factors, and inflammation: the multi-ethnic study of atherosclerosis (MESA). Invest Ophthalmol Vis Sci 2006;47:2341-2350

7. Cheung N, Rogers SL, Donaghue KC, Jenkins AJ, Tikellis G, Wong TY. Retinal 
arteriolar dilation predicts retinopathy in adolescents with type 1 diabetes. Diabetes Care 2008;31:1842-1846

8. Klein R, Klein BE, Moss SE, et al. Retinal vascular abnormalities in persons with type 1 diabetes: the Wisconsin Epidemiologic Study of Diabetic Retinopathy: XVIII. Ophthalmology 2003;110:2118-2125

9. Pemp B, Polska E, Garhofer G, BayerleEder M, Kautzky-Willer A, Schmetterer L. Retinal blood flow in type 1 diabetic patients with no or mild diabetic retinopathy during euglycemic clamp. Diabetes Care 2010;33:2038-2042

10. Sasongko MB, Wang JJ, Donaghue KC, et al. Alterations in retinal microvascular geometry in young type 1 diabetes. Diabetes Care 2010;33:1331-1336

11. Mohsin F, Craig ME, Cusumano J, et al. Discordant trends in microvascular complications in adolescents with type 1 diabetes from 1990 to 2002. Diabetes Care 2005;28:1974-1980

12. Diabetic retinopathy study. Report Number 6. Design, methods, and baseline results. Report Number 7. A modification of the Airlie House classification of diabetic retinopathy. Prepared by the Diabetic Retinopathy. Invest Ophthalmol Vis Sci 1981; 21:1-226

13. Witt N, Wong TY, Hughes AD, et al. Abnormalities of retinal microvascular structure and risk of mortality from ischemic heart disease and stroke. Hypertension 2006;47:975-981

14. Nguyen TT, Wang JJ, Islam FM, et al. Retinal arteriolar narrowing predicts incidence of diabetes: the Australian Diabetes, Obesity and Lifestyle (AusDiab) Study. Diabetes 2008;57:536-539

15. Rogers SL, Tikellis G, Cheung N, et al. Retinal arteriolar caliber predicts incident retinopathy: the Australian Diabetes, Obesity and Lifestyle (AusDiab) study. Diabetes Care 2008;31:761-763

16. McGahon MK, Dash DP, Arora A, et al. Diabetes downregulates large-conductance Ca2+-activated potassium beta 1 channel subunit in retinal arteriolar smooth muscle. Circ Res 2007;100:703-711

17. Curtis TM, Gardiner TA, Stitt AW. Microvascular lesions of diabetic retinopathy: clues towards understanding pathogenesis? Eye (Lond) 2009;23:1496-1508

18. Donaghue KC, Fung AT, Hing S, et al. The effect of prepubertal diabetes duration on diabetes. Microvascular complications in early and late adolescence. Diabetes Care 1997;20:77-80

19. Donaghue KC, Fairchild JM, Craig ME, et al. Do all prepubertal years of diabetes duration contribute equally to diabetes complications? Diabetes Care 2003;26: 1224-1229
20. Elhadd TA, Khan F, Kirk G, et al. Influence of puberty on endothelial dysfunction and oxidative stress in young patients with type 1 diabetes. Diabetes Care 1998;21:1990-1996

21. Shaw LJ, Lewis JF, Hlatky MA, et al.; National Heart, Lung and Blood Institute; American College of Cardiology Foundation. Women's Ischemic Syndrome Evaluation: current status and future research directions: report of the National Heart, Lung and Blood Institute workshop: October 2-4, 2002: Section 5: gender-related risk factors for ischemic heart disease. Circulation 2004;109: e56-e58

22. Gallego PH, Craig ME, Hing S, Donaghue KC. Role of blood pressure in development of early retinopathy in adolescents with type 1 diabetes: prospective cohort study. BMJ 2008;337:a918

23. Daniels SR, Lipman MJ, Burke MJ, Loggie JM. Determinants of retinal vascular abnormalities in children and adolescents with essential hypertension. J Hum Hypertens 1993; 7:223-228

24. Wolffsohn JS, Napper GA, Ho SM, Jaworski A, Pollard TL. Improving the description of the retinal vasculature and patient history taking for monitoring systemic hypertension. Ophthalmic Physiol Opt 2001;21:441-449 